\title{
FIRST RESULTS OF THE VLBI EXPERIMENT ON RADAR LOCATION OF THE ASTEROID 2012 DA14
}

M. Nechaeva ${ }^{1}$, A. Antipenko ${ }^{1}$, D. Bezrukov ${ }^{2}$, Vl. Bezrukovs ${ }^{2}$, A. Dementjev ${ }^{1}$,

N. Dugin ${ }^{1}$, N. Jekabsons ${ }^{2}$, R. Khutornoy ${ }^{3}$, M. Klapers ${ }^{2}$, A. Konovalenko ${ }^{4}$, V. Kulishenko ${ }^{4}$, A. Nabatov ${ }^{4}$, V. Nesteruk ${ }^{3}$, G. Pupillo ${ }^{5}$, A. Reznichenko ${ }^{4}$, E. Salerno ${ }^{5}$, I. Shmeld ${ }^{2}$, K. Skirmante ${ }^{2}$, Yu. Tikhomirov ${ }^{1}$ and V. Voytyuk ${ }^{3}$

1 Radiophysical Research Institute, B. Pecherskaya str., 25/12a, Nizhny Novgorod, 603950, Russia

2 Engineering Research Institute "Ventspils International Radioastronomy Centre”, Ventspils University College, Inženieru str., 101, Ventspils, LV-3601, Latvia

${ }^{3}$ National Space Facilities Control and Test Centre, Vityno village, Saky district, Crimea, Ukraine

${ }^{4}$ Institute of Radio Astronomy, Chervonopraporna str. 4, Kharkiv, 310002, Ukraine

5 Istituto di Radioastronomia - INAF, Via Gobetti 101, I-40129, Bologna, Italy

Received: 2013 September 3; accepted: 2013 September 17

\begin{abstract}
An international VLBI experiment on radio location of the asteroid 2012 DA14 was organized on 2013 February 15-16, during its flyby close to Earth. The purpose of observations was to investigate and specify orbital parameters of the asteroid, as well as to evaluate its rotation period and other characteristics. The irradiation of the asteroid was performed by the RT-70 transmitter at Evpatoria (Crimea, Ukraine), while the reflected signals were successfully accepted by the two $32 \mathrm{~m}$ radio telescopes at Medicina (Bologna, Italy) and Irbene (Ventspils, Latvia). Processing and interpretation of the data were performed both in the Radiophysical Research Institute at Nizhny Novgorod and in the Ventspils International Radio Astronomy Center. The first results of this experiment are presented and discussed.
\end{abstract}

Key words: instrumentation: interferometers, Very Long Baseline Interferometry methods: observational - techniques: interferometric, radar astronomy - ephemeris asteroid 2012 DA14

\section{INTRODUCTION}

The flyby of a small near-Earth asteroid 2012 DA14 at a distance of less than 30000 $\mathrm{km}$ from the Earth was one of the most significant astronomical events of 2013. The object's absolute magnitude of 24.4 suggests a diameter of $\sim 40 \mathrm{~m}$ (within a factor of two). Its close approach to the Earth provided a unique opportunity for the study of the nearby asteroids and for testing radio astronomy techniques, including the radio location method.

In addition to optical observations, several radar measurements were carried out by the Goldstone, Haystack and EISCAT observatories. The most relevant results of these 
experiments were the determination of the delay-Doppler images of the asteroid and the evaluation of its size and rotation period (Benner 2013; Vierinen 2013). According to these authors, 2012 DA14 is an elongated body with the dimensions $40 \times 20 \mathrm{~m}$ and a rotation period of more than 8 hours.

Over the past few years our group has developed a new method - the radar VLBI that uses the Very Long Baseline Interferometry technique for the radio location of nearEarth space objects. A series of radar measurements of space debris and asteroids during their close approaches to Earth have been carried out with the aim of refining their orbits (Molotov 2003, 2008; Nechaeva 2013). The flyby of asteroid 2012 DA14, which occurred in February of 2013, allowed to make a detailed analysis of the reflected echo, particularly with regard to the measurements of Doppler shift frequency.

The radar VLBI method (Alekseev 2000; Nechaeva 2007) is a radar technique that has been applied since 2001 to determine the path of planets, asteroids and space debris objects. This technique combines the advantages of the radar measurements with the VLBI techniques. In particular, while radars can provide accurate measurements of range and radial velocity, the VLBI correlation gives precise information on the angle and angular rate of the observed object. With this technique it is possible to determine the exact position of near-Earth objects (complete solution of the navigation task).

\section{THE EXPERIMENT}

The international radar VLBI experiment on radio location of asteroid 2012 DA14 was carried out on 2013 February 15-16. On this occasion three radar sessions were performed at the time of the asteroid's closest approach $(33000 \mathrm{~km})$. Three additional sessions were later performed when its distance from the Earth has increased up to 10 times. The time duration of each session ranged from 20 to $45 \mathrm{~min}$. The ephemeris of the asteroid, and consequently the coordinates for the antennas pointing, were calculated by using the web-interface to JPL's HORIZONS system (http://ssd.jpl.nasa.gov). We have used JPL's Horizons solution \#49. In order to improve the accuracy of the antennas pointing, the precise calibration measurements were performed between each radar session by observing natural radio sources. The calibration sources were characterized by flux densities greater than $2 \mathrm{Jy}$ and were located within an angular distance of $20^{\circ}$ from the asteroid during the observation.

Figure 1 illustrates a schematic diagram of the experiment. The radio location of the asteroid was carried out by the RT-70 transmitter at Evpatoria (Ukraine, Crimea). Irradiation was performed in monochromatic mode at a frequency of $5 \mathrm{GHz}$, in linear-frequencymodulated (LFM) mode, with a frequency deviation of $512 \mathrm{kHz}$ and a modulation period of 4,16 and $32 \mathrm{~ms}$ in different sessions. The transmitting power during experiment was approximately $70 \mathrm{~kW}$.

The reception of the reflected signals was implemented by the $32 \mathrm{~m}$ radio telescopes at Medicina (Italy) and Irbene (Ventspils, Latvia). Signals were recorded in a bandwidth of $2 \mathrm{MHz}$ by the acquisition systems TN16, MK5B at Irbene and by MK5A at Medicina. During the first radar session the Doppler shift frequency reached a value of $-60 \mathrm{kHz}$ at the radial velocity of the asteroid $\sim 2 \mathrm{~km} / \mathrm{s}$. During the last session, the measured shift was $-210 \mathrm{kHz}$ at the radial velocity $\sim 6 \mathrm{~km} / \mathrm{s}$. The reflected echoes were successfully detected by both radio telescopes in all radar sessions. 


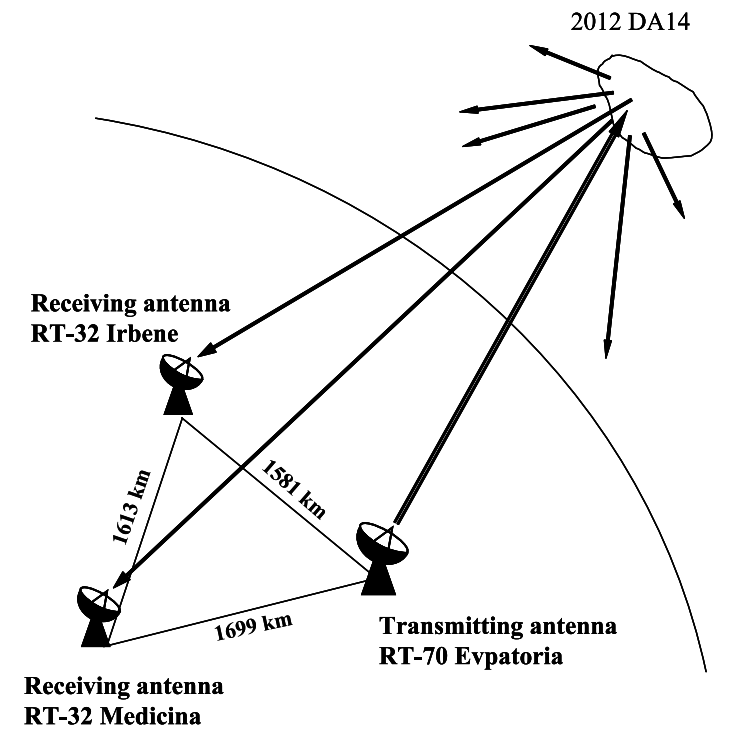

Fig. 1. Schematic diagram of the radar VLBI experiment on radio location of the near-Earth asteroid 2012 DA14.

\section{RESULTS}

The processing of collected data was carried out at the Radiophysical Research Institute (Nizhny Novgorod) by using the NIRFI-4 correlator, originally designed for VLBI data processing. The data were also processed by the correlator at the International Radio Astronomy Center (Ventspils), currently being developed for radar studies of space debris. The asteroid coordinates provided by the JPL web-service HORIZONS were used for the calculation of the parameters necessary to data processing. The functions describing the time dependences of delay and Doppler frequency shift, were then derived. Finally, these functions were used for a preliminary signal processing and a further correlation and spectral analyses.

The data processing was performed in three stages: (1) autocorrelation of the signals recorded in each receiving station; (2) cross-correlation between the signal transmitted by the radar and the reflected signal received by each antenna (bi-static mode); (3) crosscorrelation of the signals recorded by the receiving stations (VLBI-mode).

The first step of the data processing - autocorrelation of the received echo-signals and further spectral analysis - was implemented during the observations and before both the cross-correlation processing for selective check of records and the detection of echosignals. An example of autocorrelation spectrum of the signals recorded at Irbene, is given in Figure 2. In this graph, echoes coming from the asteroid were clearly detected at different times with high $\mathrm{S} / \mathrm{N}$ ratios.

As expected, the echo frequencies changed in time, due to asteroid's movement

The time dependence of the amplitude of autocorrelation spectra has been studied at different time intervals, with the purpose of evaluating the rotation period and size of the asteroid. Unfortunately, the estimation of this parameter could not be finally performed, since the continuous observations were shorter than the rotation period of the asteroid. Figures 3 and 4 show the dynamic autocorrelation spectra, derived from data collected at Medicina and Irbene, respectively. 


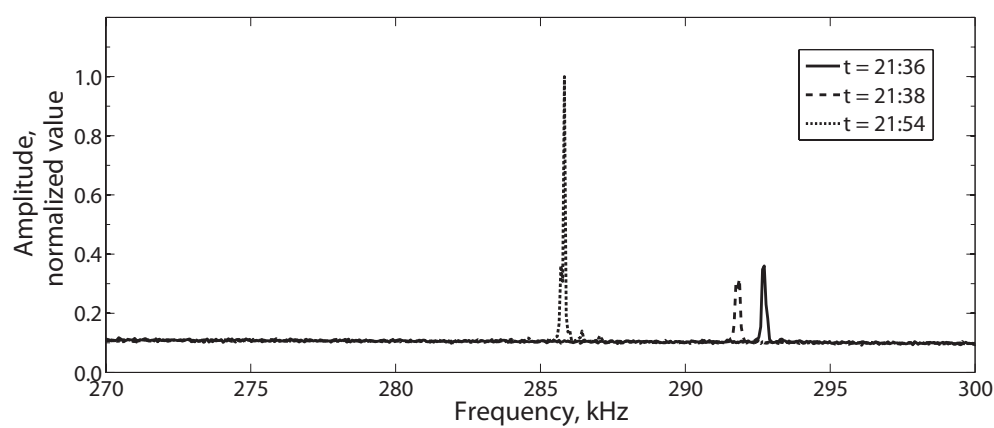

Fig. 2. The autocorrelation spectra calculated at 21:36 UT (solid line), 21:38 UT (dashed line) and 21:54 UT (dotted line). Integration time: 0.016 s. Irbene, 2013 February 15.

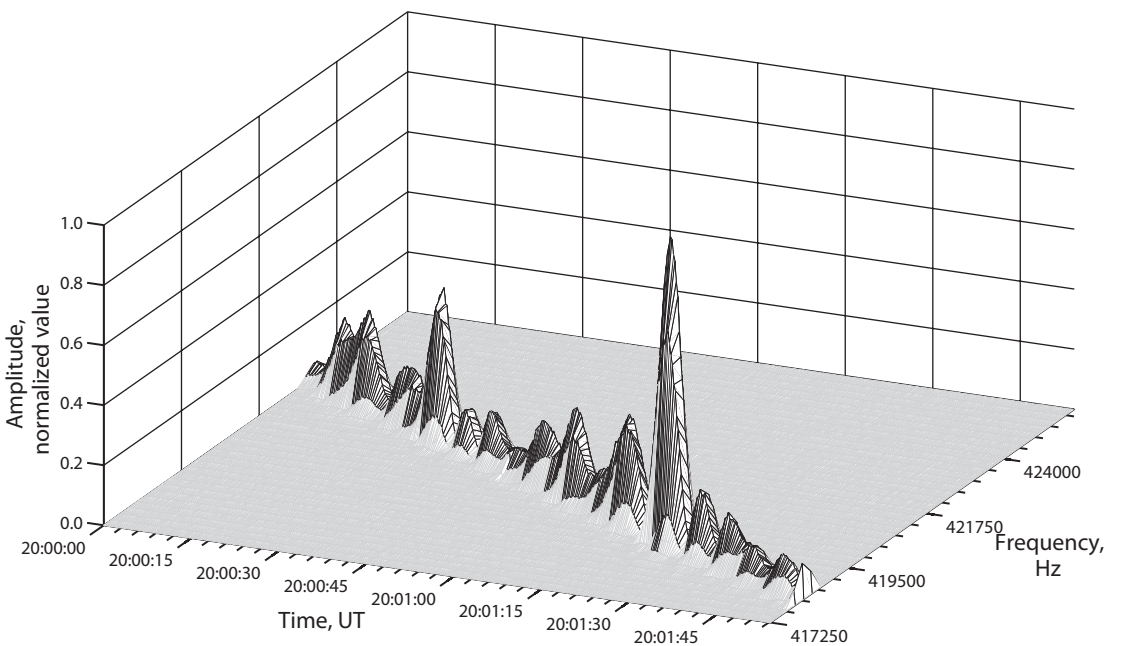

Fig. 3. The amplitude of power spectrum as the function of time and frequency. Integration time: 0.25 s. Medicina, 2013 February 15.

These spectra were obtained during the first radar session, i.e. when the asteroid was at its minimum distance from Earth. We find that the maximum signal amplitude did not occur at exactly the same time at the two VLBI stations. This may be due to the fact that the asteroid was visible by the two antennas under different view angles and therefore the reflected signal, coming from different regions of the asteroid surface, arrived at the two antennas in different times. The quasi-periodic character of the signal amplitude is associated with interferences coming from a few bright areas on the asteroid surface.

The second step of data processing consisted in performing the cross-correlation between the transmitted signal and the reflected echoes received by the antennas at Irbene and Medicina. Transmitted and received signals were multiplied by time delay and frequency offset. Resulting signals underwent spectral analysis. An example of crosscorrelated spectrum is given in Figure 5. The frequency of spectral maximum corresponds to the Doppler shift which arises on the path "transmitter - asteroid - receiver" and is determined by the radial velocity of the object.

The third step of data processing consisted in performing the cross-correlation of the 


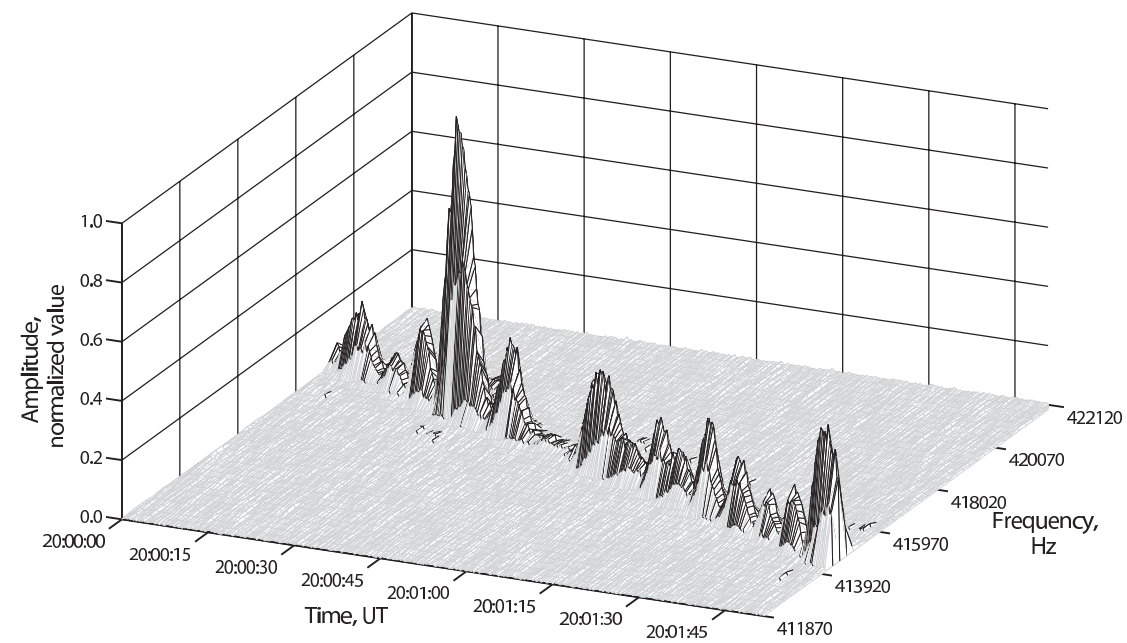

Fig. 4. The amplitude of power spectrum as the function of time and frequency. Integration time: 0.25 s. Irbene, 2013 February 15.

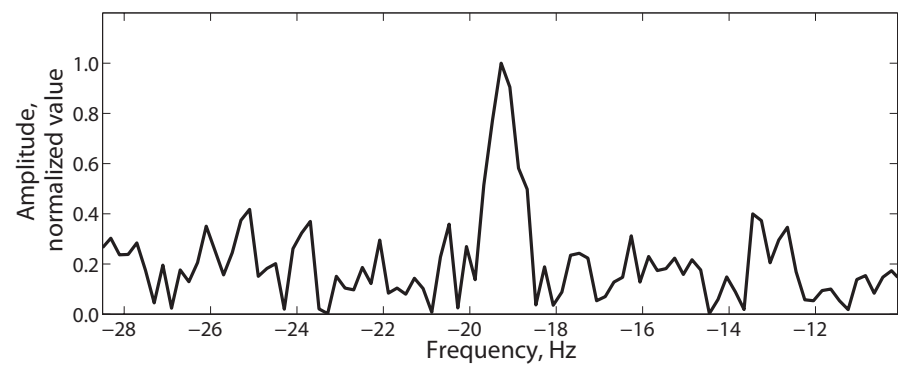

Fig. 5. The spectrum of signal derived from the correlation between the signal transmitted by the Evpatoria antenna and reflected echo recorded at Irbene. Horizontal axis represents the difference between the measured Doppler frequency shift and the frequency derived from the preliminarily known data. Integration time was 5 s, observed on 2013 February 16, 04:53:02 UT.

echo-signals received by antennas. This is the basic procedure for correlation of the VLBI astronomical data. The further spectral analysis allowed the determination of the interferometer response and the measurement of the interference frequency in correspondence with the spectral maximum peak. The interference frequency was determined by the rate of change of the distances between the observed object and the receiving antennas.

An example of power spectrum calculated at this stage of the process is shown in Figure 6. The measured frequencies allow to determine the angular velocity of the asteroid if the range and radial velocity are known.

\section{CONCLUSIONS}

The described radar experiment on the asteroid 2012 DA14 can be considered as one of the most successful observations of asteroids performed by our group. The large size of the asteroid, together with its close distance from Earth at the time of observation, allowed us to clearly detect the reflected echoes with a high level of details. The first results of the data processing revealed the detection of a powerful echo in the first three radar 


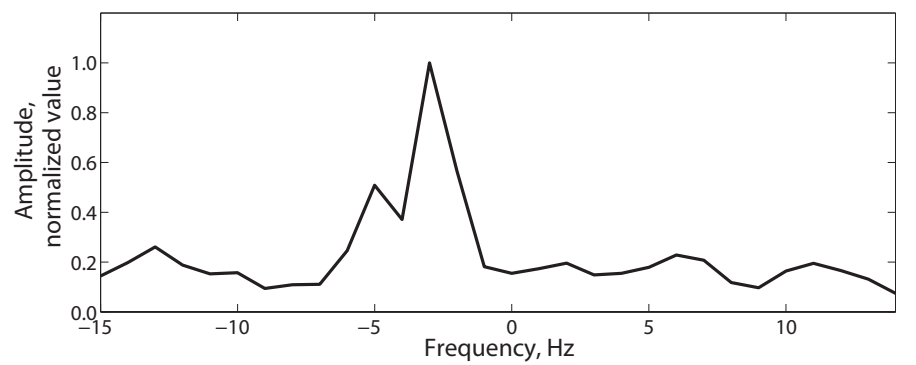

Fig. 6. The spectrum of signal as a result of the correlation of the echoes received by the antennas at Irbene and Medicina. Integration time was 1 s, observed on 2013 February 15, 20:01:24 UT.

sessions, when the asteroid was at short distance from Earth. A weaker signal was also detected when the distance of 2012 DA14 from the Earth has increased up to 10 times. The measurements of the Doppler shift frequency were successfully carried out during whole time of the radar experiment. Furthermore, the detection of the interferometer response from an asteroid in the VLBI-mode was also achieved for the first time.

Ongoing activities include the processing of the linear frequency modulated signal trying to measure the delay on cross-correlation of the two received signals and, consequently, to evaluate the length of signal propagation path and the angular coordinates of the asteroid. Thus, as soon as the data processing is finished, the parameters for the complete solution of the navigation task (estimation of the distance, radial velocity, angular velocity and angular coordinates) will be provided.

ACKNOWLEDGMENTS. This contribution was partially supported by the State Space Agency of Ukraine (project "Science - Interferometer"), Russian Foundation of Basic Research (project 13-02-00586-a) and Ventspils City Council Financed Project "Research in VIRAC using the IRBENE radio telescope". The publication of this article is supported by the European Regional Development Fund project "International competitiveness and capacity-building of satellite research" (SATTEH,

No.2010/0189/2DP/2.1.1.2.0/10/APIA/VIAA/019).

\section{REFERENCES}

Alekseev V. A., Lipatov B. N., Reznikova V. A. 2000, Radiophysics and Quantum Electronics, 43, No. 8, 607

Benner L. 2013, http://echo.jpl.nasa.gov/asteroids/2012DA14/ 2012DA14_planning.html; http://www.youtube.com/watch?v=o-8qNuUyO-4

Molotov I., Kovalenko A., Samodurov V. et al. 2003, Astron. and Astrophys. Transactions, 22, Nos. 4-5, 743

Molotov I., Nechaeva M., Falkovich I. et al. 2008, in Measuring the Future, Proceedings of the 5th IVS General Meeting, eds. A. Finkelstein \& D. Behrend, Nauka Publishers, p. 30

Nechaeva M. B., Antipenko A. A., Dement'ev A. F. et al. 2007, Radiophysics and Quantum Electronics, 50, No. 7, 527

Nechaeva M., Antipenko A., Bezrukovs V. et al. 2013, Baltic Astronomy, 22, 35

Vierinen J. 2013. http://kaira.sgo.fi/2013_02_01_archive.html 